\title{
A Method for Comparing the Performance of Power Plants for Vertical Flight
}

\author{
H. S. 'TSIEN ${ }^{1}$
}

Daniel and Florence Guggenheim Jet Propulsion Center, California Institute of Technology, Pasadena, Calif.

\begin{abstract}
A new method of power plant selection for vertical flight is proposed. It can be used to determine whether the performance of a rocket design can be improved by substituting for the rocket motor a different power plant such as a ramjet. Calculations indicate that there are advantages in using the ramjet provided the power plant ean be made to operate under rapid acceleration and at high altitudes.
\end{abstract}

$\mathbf{A}$ PROBLEM constantly facing engineers who use jet propulsion power plants is to determine the best power plant among a multitude of possible power plants for a particular design application. A very general method of power plant selection was proposed, perhaps for the first time, by W. Bollay and E. Redding, based upon the concept of lowest total installation weight. The total installation weight for a specified thrust at given altitude and speed of flight is the sum of dry power plant weight plus the weight of fuel and fuel tank for a given duration of flight at that altitude and speed. The fuel and fuel tank weight increase with the increase in flight duration. Thus, a light power plant with large fuel consumption, such as a rocket, is competitive with a heavier power plant with smaller fuel consumption only at short flight durations. This concept was extensively developed by Th. von Kármán in his general analysis of jet propulsion power plants (1). ${ }^{2}$ This method of power plant selection was also described by A. L. Lowell (2).

However, no actual vehicle will fly at constant speed and constant altitude. There is always a definite flight plan describing the speed and altitude as functions of time. Hence, true power plant selection must depend upon a sort of weighted average of different speeds, and altitudes according to the particular flight plan of the vehicle under consideration. If the speed and altitude of the vehicle are rapidly varying, as in the case of accelerated vertical flight, then the selection based upon total installation weight at a fixed speed and a fixed altitude would be quite wrong. The purpose of the present note is to give a different method of power plant selection for vehicles in vertical flight. A meteorological sounding vehicle is a direct example of such an application. For other types of vehicle, the vertical powered flight is often a good approximation to the true flight trajectory during the application of propulsive power. Therefore the proposed method is believed

Received January 3, 1952.

${ }^{1}$ Robert H. Goddard Professor of Jet Propulsion.

${ }^{2}$ Numbers in parentheses refer to the References on page 212. to have a wider range of usefulness than is, perhaps, first apparent.

\section{General Relation}

It will be assumed in this analysis that the gravitational acceleration and the effective exhaust velocity of the power plant are constants. For moderate altitudes, the decrease of the gravitational acceleration from its sea-level value is very small and thus negligible. The effective exhaust velocity of the power plant, defined as ratio of thrust generated by the power plant to the mass rate of consumption of fuel or propellant carried within the vehicle, is of course a variable, not a constant, even for a given power plant using a given fuel or propellant. For a rocket, this variation is caused by the change in atmospheric pressure with altitude, and is usually small enough to be neglected. For a ramjet, the effective exhaust velocity increases very rapidly with speed of the vehicle in the subsonic range, but in the supersonic range the exhaust velocity is again almost constant with respect to the speed of the vehicle, within the useful speed range of the engine (3). For other power plants, the variation of the effective exhaust velocity may be more complicated; but as a first approximation, it is generally possible to use an average value as the assumed constant exhaust velocity.

For a rocket vehicle, the air drag is proportional to the cross-sectional area of the body, but the mass of the vehicle is proportional to the volume of the body. Therefore for vehicles of similar design, the air drag is proportional to the square of the body diameter, while the mass of the vehicle is proportional to the cube of the body diameter. For large rocket vehicles the drag force is then negligible with respect to the gravitational force. Calculations seem to show that for a high performance rocket of 50 tons gross weight, the air drag reduces the velocity at the end of powered flight by only 5 per cent. Hence for a large rocket vehicle, the effects of air drag on the performance can be neglected in the approximate analysis attempted here. When another power plant is used, the design cannot perhaps be made as compact as the rocket. For instance, the ramjet requires a rather large duct to produce a sizable thrust. Then the air drag may not be negligible even for a large vehicle. However, in such cases the air drag of the power plant installation can be charged against the thrust produced. As an 
example, the air drag of the outside surfaces of the ramjet duct can be deducted from the thrust, produced by the ramjet, and the power plant is considered to produce a smaller "net thrust" while the body drag is considered to remain at the same magnitude without the ramjet duct. Therefore if one takes the effective, exhaust velocity as that based upon the net thrust, then the same argument for a large rocket applies and the drag of the body can be neglected for large vehicles.

For vehicles that are not so large, neglect of the air drag will certainly introduce an error. But the emphasis here is the comparison of the performance obtainable from different power plants rather than the absolute value of performance. Therefore the error made in this way is believed to be not large, and the method given below is useful even for moderate-sized vehicles.

Let $m$ be the mass of the vehicle at time instant $t$ when the vertical position and vertical velocity of the vehicle are $y(t)$ and $\dot{y}(t)$, respectively. Denote by $c$ and $g$ the constant exhaust velocity and the gravitational acceleration. Then the balance of inertia force and gravitational against the thrust (Fig. 1) gives

$c \frac{d m}{d t}+m\left(\frac{d \dot{y}}{d t}+g\right)=0$.

If the initial velocity at $t=0$ is zero, as is usually the case, Equation [1] integrates to

$\log \frac{m}{m_{0}}=-\frac{1}{c}(\dot{y}+g t) \ldots \ldots$

If the subscript 1 denotes conditions at the end of the powered flight, then Equation $[2]$ gives

$\log \frac{m_{0}}{m_{1}}=\frac{1}{c}\left(\dot{y}_{1}+g t_{1}\right)$

This is the fundamental performance equation for vertical flight. It has been derived previously by many authors (4), but the present deviation clearly shows that it is quite general and independent of the particular way the thrust is programmed during the powered flight.

For a vehicle intended for obtaining long range but having a vertical powered trajectory, the performance is essentially determined by the velocity at the end of powered flight or $\dot{y}_{1}$. For an atmospheric sounding vehicle, the summit altitude is determined by $y_{1}$ and $\dot{y}_{1}$. The two vehicles having the similar thrust programming during FORCES ON A VEHICLE IN VERTICAI ASCENT
FIG. 1. BALANCE OF

powered flight and the same $\dot{y}_{1}$ and $t_{1}$ will have the same $y_{1}$ and $\dot{y}_{1}$. To simplify the calculation, the performances of two vehicles with different power plants are made to be the same by specifying that the values of $y_{1}, \dot{y}_{1}$, and $t_{1}$ at end of the powered flight are the same for the two vehicles. This condition will be satisfied if the acceleration programs of the two vehicles are the same. The thrust programs of the two vehicles are different, however, due to the different fuel consumption of the two power plants. Therefore, one of the criteria for equal performance is the value of $\dot{y}_{1}+g t_{1}$, occurring on the right of Equation [3]. According to Equation [3], then, to have the same performance, $c \log \left(m_{0} / m_{1}\right)$ must be the same for vehicles. This conditions will be used to compare the performance of different power plants.

Now let $w_{0}, w_{s}, w_{e}$, and $w_{1}$ denote the gross weight, the weight of structures and pay load, the engine weight, and the weight of the vehicle, respectively, at the end of powered flight. Then

$$
w_{1}=w_{s}+w_{e} \ldots \ldots \ldots \ldots \ldots[4]
$$

Equation [3] can be written as

$$
c \log \frac{w_{0}}{w_{s}+w_{e}}=\dot{y}_{1}+g t_{1} .
$$

Consider now two vehicles, both having the same gross weight $w_{0}$; one is a rocket with $c=c^{*}, w_{s}=w_{s}{ }^{*}$, $w_{e}=w_{e}{ }^{*}$, and

$$
\frac{w_{0}^{*}}{w_{s}^{*}+w_{e}^{*}}=\frac{1}{1-\zeta}
$$

where $\zeta$ is the so-called propellant loading ratio or the fraction of propellant weight in the gross weight. The other vehicle with $w_{0}=w_{0}{ }^{*}, w_{s}=w_{s}{ }^{*}$, but with $c$ and $w_{e}$ different from $c^{*}$ and $w_{e}{ }^{*}$. This means that the structural weight and pay load of the vehicle with the alternate power plant remain the same as the rocket, but the engine weight is different. To compare the performance of the power plants, the maximum allowable engine weight $w_{e}$ for equal performance should be calculated. If the actual engine weight is less than this calculated maximum, then the pay load can be increased over that possible for the rocket. In other words, the rocket motor can be substituted by the new power plant with a net gain in performance. If the actual engine weight is higher than the calculated maximum, then the alternate power plant will give a poorer performance than the rocket engine.

The conditions to determine the maximum allowable engine weight $w_{e}$ are

$$
\begin{aligned}
& c^{*} \log \frac{1}{1-\zeta}=c^{*} \log \frac{w_{0}^{*}}{w_{s}^{*}+w_{e}^{*}}=\dot{y}_{1}+g t_{1} \\
& c \log \frac{w_{0}}{w_{s}+w_{e}}=c \log \frac{w_{0}^{*}}{w_{s}^{*}+w_{e}}=\dot{y}_{1}+g t_{1} \ldots \ldots
\end{aligned}
$$

Therefore by eliminating $\dot{y}_{1}$ and $t_{1}$, one has

$$
\frac{w_{e}}{w_{e}{ }^{*}}-1=\left(\frac{w_{1}^{*}}{w_{e}^{*}}\right)\left[(1-\zeta)^{-\left(1-\left(c^{*} / c\right)\right)}-1\right] .
$$

If instead of the effective exhaust velocity, the engine consumption is specified by the specific consumption $s$ 
in $\mathrm{lb}$ of fuel per $\mathrm{lb}$ of thrust per unit time, then, since $s$ is inversely proportional to $c$, Equation [8] can be written as

$$
\frac{w_{e}}{w_{e}{ }^{*}}-1=\left(\frac{w_{1}^{*}}{w_{e}{ }^{*}}\right)\left[(1-\zeta)^{-\left(1-\left(s / s^{*}\right)\right)}-1\right] \ldots[\text { [9] }
$$

where $s$ is the specific consumption of the engine under investigation and $s^{*}$ the specific consumption of the comparison rocket engine. The left sides of Equations [8] and [9] are the maximum allowed increase in engine weight over the rocket engine weight divided by the rocket engine weight. The first factors on the right sides of Equations [8] and [9] are the ratio for the rocket vehicle of the weight at end of powered flight or burnout and the engine weight. The second factor on the right sides of Equations [8] and [9] are plotted in Fig. 2.

Fig. 2 shows that as the propellant loading ratio $\zeta$ for the rocket vehicle increases, the allowable increase in engine weight for a fixed reduction in specific consumption $s$ is extended. Thus there is wider latitude in the choice of power plant for high performance vehicles than there is for a low-performance vehicle. This is certainly an encouraging fact for design engineers. It also points out the fact that the choice of power plant cannot be made independent of the performance of the vehicle but rather is intimately related to the performance of the vehicle.

\section{Applications}

As a first example, consider the possibility of using

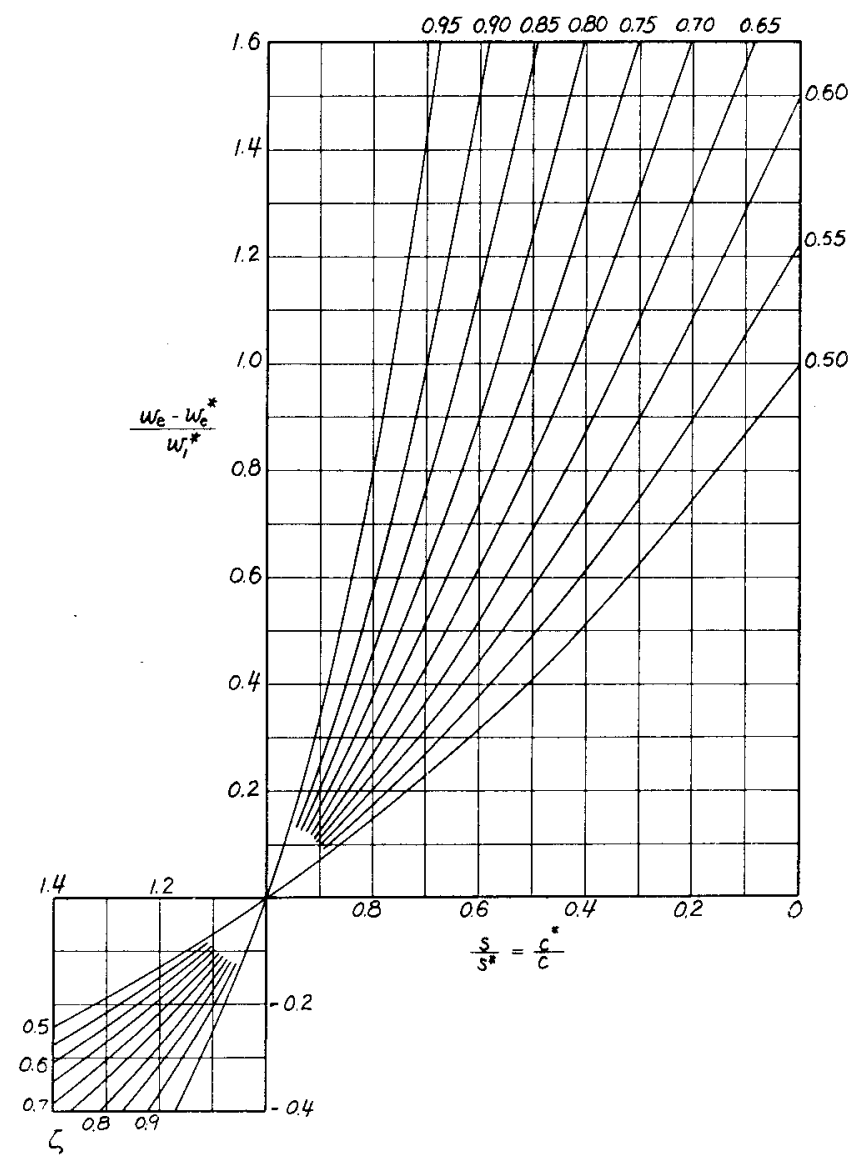

FIG. 2. ENGINE WEIGHT FACTOR AS FLNCTIONS OF ROCKET PROPELLANT LOADING RATIO $\zeta$ AND SPECIFIC FUEL CONSUMPTION $s$. CF. EQUATIONS [8] AND [9] a ramjet as the propulsive power plant for a vehicle of the performance of a V-2 rocket. The weight breakdown of a V-2 is given in Table 1 (5):

\section{Table 1 V-2 Rocket}

\begin{tabular}{|c|c|c|c|}
\hline \\
\hline Explosive charge & $980 \mathrm{~kg}$ & & \\
\hline \multirow[t]{2}{*}{ Auxiliary devices } & $300 \mathrm{~kg}$ & & \\
\hline & & $1280 \mathrm{~kg}$ & \\
\hline Structural Engine & & $1750 \mathrm{~kg}$ & \\
\hline Pumping unit & $450 \mathrm{~kg}$ & & \\
\hline \multirow[t]{2}{*}{ Rocket motor } & $550 \mathrm{~kg}$ & & \\
\hline & & $1000 \mathrm{~kg}$ & \\
\hline Weight at burn-out & & & $4,030 \mathrm{~kg}$ \\
\hline \multirow{4}{*}{$\begin{array}{l}\text { Propellant and Fuel: } \\
\text { Propellant } \\
\text { Fuel for turbine }\end{array}$} & & & \\
\hline & & $8750 \mathrm{~kg}$ & \\
\hline & & $200 \mathrm{~kg}$ & \\
\hline & & & $8,950 \mathrm{~kg}$ \\
\hline Gross weight & & & $12,980 \mathrm{~kg}$ \\
\hline
\end{tabular}

Therefore $\zeta=8950 / 12,980=0.69$. The ratio $w_{1}{ }^{*} / w_{e}{ }^{*}$ $=4030 / 1000=4.03$. The effect exhaust velocity including the fuel for turbine drive can be taken as $c^{*}=7000 \mathrm{ft} / \mathrm{sec}$ or $s^{*}=16.56 \mathrm{lb}$ per lb thrust per hr.

For a ramjet, the fuel consumption based upon net thrust can be taken as $s=4 \mathrm{lb}$ per lb thrust per $\mathrm{hr}$ (3). Then, by using Fig. 2, the maximum allowable increase in engine weight is given by

$$
\frac{w_{e}}{w_{e}^{*}}=6.77
$$

This means that the substitute ramjet engine could weigh as much as $14,900 \mathrm{lb}$ without impairing the performance of the V-2. The V-2 rocket has a thrust of $55,000 \mathrm{lb}$. This is also the average thrust to be produced by the ramjet. Then the maximum allowable ramjet weight is $0.271 \mathrm{lb}$ per $\mathrm{lb}$ of thrust. The actual ramjet weight is probably less than this value. Therefore if the ramjet can be made to operate properly under conditions of rapid vertical acceleration and altitude variation up to high altitude, then there is advantage in substituting the ramjet for the rocket engine. This possibility has motivated more accurate calculations of a ramjet in vertical flights by L. H. Schindel (6) and J. V. Rowny (7). A ramjet as the power plant for the first stage of a high performance seems particularly attractive.

There are studies $(8,9)$ on the possibility of using the nuclear reactor as an energy source for rocket propulsion. In such a nuclear rocket the reactor may take the form of porous material through which the working fluid, say hydrogen, is passed and heated. A recent investigation by L. Green (10) indicated the feasibility of such a scheme. Now one of the difficulties of using nuclear reactor is the heavy weight of the reactor, particularly when a radiation shield is required. Counteracting the weight is the higher effective exhaust velocity possible. For instance, if hydrogen could be heated to $6000 \mathrm{~F}$, then the data given by F. J. Malina and M. Summerfield (11) point to a value of $c$ equal to 26,000 $\mathrm{ft} / \mathrm{sec}$ or $s=4.46 \mathrm{lb}$ per $\mathrm{lb}$ of thrust per $\mathrm{hr}$. This 
aspect of the problem of a nuclear rocket can, however, be analyzed by using the present method. For example, if the high performance rocket with energetic chemical propellant has the following specifications.

$$
\begin{array}{ll}
\zeta & =0.80 \\
s^{*} & =10 \mathrm{lb} \text { per } \mathrm{lb} \text { of thrust per } \mathrm{hr} \\
w_{1}{ }^{*} / w_{e}{ }^{*} & =2.7
\end{array}
$$

Then according to Equation [91, a nuclear rocket engine giving performance equal to the chemical rocket can have an engine weight 4.89 times the chemical rocket motor. In other words, if the nuclear reactor together with its necessary auxiliary construction increases the engine weight by more than 389 per cent, then the nuclear rocket is not feasible for the performance studied. If the increase in weight is not so much, then the nuclear rocket is worth while.

\section{Detail Improvement of Rocket Engine}

A rocket designer is often confronted with the problem of improving a given design at the expense of increasing the engine weight. For example, the effective exhaust velocity of a propellant can be generally increased by increasing the combustion chamber pressure. But increasing the chamber pressure would require heavier construction and an increase in the feed pressure for the propellant. Increasing the feed pressure would in turn increase the weight of the feed system. Therefore the improvement in propellant consumption is to be achieved only with higher engine weight. The present analysis can be used to determine whether such a change will or will not improve the overall performance of the vehicle.

For small changes in effective exhaust velocity or specific consumption, Equations [8] and [9] can be simplified to relate the allowable fractional increase in engine weight $\Delta w_{e} / w_{e}{ }^{*}$ to fractional increase in effective exhaust velocity $\Delta c / c^{*}$ or specific consumption $\Delta s / s^{*}$. Thus, taking only the first order terms, one has from Equations [8] and [9].

$$
\frac{\Delta w_{e}}{w_{e}^{*}}=\frac{\Delta c}{c^{*}}\left(\frac{w_{1}^{*}}{w_{e}^{*}}\right) \log \left(\frac{1}{1-\zeta}\right) \ldots \ldots \ldots[10]
$$

and

$$
\frac{\Delta w_{e}}{w_{e}^{*}}=\frac{\Delta s}{s^{*}}\left(\frac{w_{1}^{*}}{w_{e}^{*}}\right) \log (1-\zeta)
$$

These equations give the allowable fractional increase in engine weight for equal performance. If the actual increase in engine weight is less, then the modification in design improves performance, and is thus desirable. If the actual increase is more, the modification is not practical.

By taking the $\mathrm{V}-2$ as an example, it can be easily calculated that 1 per cent improvement in consumption is worth 4.72 per cent increase in engine weight.

\section{References}

1 "Comparative Study of Jet Propulsion Systems as Applied to Missiles and Transonic Aircraft," by Th. von Kármán, Memorandum No. JPL-2, 1944, Jet Propulsion Laboratory, California Institute of Technology.

2 "A Guide to Aircraft Power Plant Selection," Aeronautical Engineering Review, April 1947, pp. 22-25.

3 The theoretical performance of ramjet as calculated by many authors; e.g., "The Gas Dynamic Theory of the Ramjet," by J. Reid, Aeronautical Research Council, London, Report \& Memorandum. No. 2370, 1950.

4 See, for instance, "Flight Anaylsis of the Sounding Rocket," by F. J. Malina and A. M. O. Smith, Journal of the Aeronautical Sciences, vol. 5, 1932, pp. 199-202.

5 "Ballisties of the Future," by J. M. J. Kooy and J. W. H. Uytenbogart, McGraw-Hill Book. Co., Inc., New York, 1946, p. 297.

6 "Application of Ramjet to High Altitude Sounding Vehicle," by L. H. Schindel, M.S. thesis (Aeronautical Engineer), Massachusetts Institute of Technology, 1948.

7 "Application of Ramjet to Vertical Ascent," by J. V. Rowny, thesis (Aeronautical Engineer), California Institute of Technology, 1949.

8 "The Atomic Rocket," by J. R. Shepherd and A. V. Cleaver, Journal of the British Interplanetary Society, vol. 7, 1949, pp. 185-194, 234-241.

9 "Rockets and Other Thermal Jets Using Nuclear Energy," by H. S. Tsien, in "The Science and Engineering of Nuclear Power," Addison-Wesley Press, Inc., Cambridge, Mass., vol. II, 1949 , p. 124.

10 "Gas Cooling of a Porous Heat Source," by L. Green, Jr., Journal of Applied Mechanics, vol. 19, June 1952, pp. 173-178.

11 "The Problem of Escape From the Earth by Rocket," by F. J. Malina and M. Summerfield, Journal of the Aeronautical Sciences, vol. 14, 1947, pp. 471-480, particularly Table 2. 